\title{
Correlation between multi-drug resistant organisms and antimicrobial use among in-hospital patients at a tertiary hospital in the Philippines from July 2010 to June 2014
}

\author{
RK So,, MM Mendoza \\ From 3rd International Conference on Prevention and Infection Control (ICPIC 2015) \\ Geneva, Switzerland. 16-19 June 2015
}

\section{Introduction}

Infections caused by multidrug resistant organisms (MDRO) are associated with higher morbidity, mortality and healthcare costs. WHO recommends hospitals to monitor antimicrobial use to reduce MDRO prevalence.

\section{Objectives}

This 4-year study described the annual MDRO prevalence and annual antimicrobial consumption. It also investigated their relationship.

\section{Methods}

Annual antibiogram of Methicillin Resistant S.aureus (MRSA), ESBL E.coli, ESBL K.pneumoniae, and MDR $P$. aeruginosa were evaluated. Data on annual consumption (Defined Daily Dose) of selected antimicrobials were analyzed. Linear regression was used to analyze trend in antimicrobial consumption and MDRO prevalence. Pearson's correlation coefficient was used to determine their relationship. A p-value $<0.05$ and $\mathrm{r}^{2}>0.5$ were considered statistically significant.

\section{Results}

There was a significant increase in annual patient days while annual antibiotic usage decreased. The most common antibiotic class used was cephalosporin, followed by beta-lactam/beta-lactamase inhibitors then fluoroquinolones. Individually, piperacillin-tazobactam, ceftriaxone and ertapenem use significantly increased. The prevalence of ESBL E.coli significantly increased, ESBL K.pneumoniae and MRSA remained stable and MDR
$P$. aeruginosa significantly decreased. The increased consumption of cefazolin, cefepime, meropenem and cotrimoxazole were positively correlated with increased ESBL E.coli prevalence. The higher use of antimicrobials without anti-Pseudomonal activity ceftriaxone and ertapenem versus piperacillin-tazobactam were positively correlated with decreased MDR P.aeruginosa prevalence. MRSA prevalence positively correlated and mirrored linezolid usage as it is a $2^{\text {nd }}$ line agent for it. The absence of increase in ESBL K.pneumoniae prevalence may be due to decreased fluoroquinolone use.

\section{Conclusion}

This study at our institution found that antimicrobial use did not increase despite increase in annual patient days. The prevalence of ESBL E.coli increased, ESBL $K$. pneumoniae and MRSA remained stable; MDR $P$. aeruginosa decreased. A positive correlation between antimicrobial use and MDRO prevalence was established. Establishing an antibiotic restriction program is recommended to address the significant prevalence of MDRO.

\section{Disclosure of interest \\ None declared.}

Published: 16 June 2015

\section{doi:10.1186/2047-2994-4-S1-P171}

Cite this article as: So and Mendoza: Correlation between multi-drug resistant organisms and antimicrobial use among in-hospital patients at a tertiary hospital in the Philippines from July 2010 to June 2014. Antimicrobial Resistance and Infection Control 2015 4(Suppl 1):P171. 